\title{
Estructura genética de un grupo de capibaras, Hydrochoerus hydrochaeris (Rodentia: Hydrocheridae) en los Llanos orientales colombianos
}

\author{
Adriana Maldonado-Chaparro ${ }^{1}$, Luz Mery Bernal-Parra ${ }^{1}$, Gustavo Forero-Acosta ${ }^{1}$ \\ \& Manuel Ruiz-Garcia ${ }^{2 *}$ \\ 1. Escuela de Ciencias Agrícolas Pecuarias y del Medio Ambiente, Universidad Nacional Abierta y a Distancia. Bogotá \\ DC., Colombia; maldonado.aa@gmail.com,lmbernalp@gmail.com, gforero@unad.edu.co \\ 2. Laboratorio de Genética de Poblaciones Molecular-Biología Evolutiva. Departamento de Biología. Facultad de \\ Ciencias. Pontificia Universidad Javeriana. Cra 7 $73-82$. Bogotá DC., Colombia; mruizgar@yahoo.es, \\ mruiz@javeriana.edu.co \\ * Correspondencia
}

Recibido 17-VIII-2010. Corregido 10-II-2011. Aceptado 11-III-2011.

\begin{abstract}
Genetic structure of a group of capybaras, Hydrochoerus hydrochaeris (Rodentia: Hydrocheridae) in the Colombian Eastern Llanos. The capybaras are the biggest rodents in the world but, however, there are not extensive population genetics studies on them. In the current work, we studied the genetic structure of a troop of 31 capybaras (Hydrochoerus hydrochaeris) sampled in Hato Corozal, Casanare Department at the Colombian Eastern Llanos, by means of five microsatellite markers. The gene diversity was 0.61 and the average allele number was 5.2, which is a medium-low level for markers of this nature. Out five markers employed, three were in Hardy-Weinberg equilibrium meanwhile one showed a significant homozygote excess and other presented a significant heterozygote excess. There were not significant genetic differences between males and females inside this troop. The application of different procedures to determine possible historical demographic changes (population expansions or bottlenecks) clearly showed that the population analyzed crossed over a very narrow recent bottleneck. The illegal hunt is the possibly cause of this strong genetic bottleneck. Rev. Biol. Trop. 59 (4): 1777-1793. Epub 2011 December 01.
\end{abstract}

Key words: capybara, Hydrochoerus hydrochaeris, DNA microsatellites, genetic structure, bottleneck, Colombian Eastern Llanos.

El capibara, chigüiro o carpincho (Hydrochoerus hydrochaeris, Lineaus 1766) es posiblemente la única especie sobreviviente de la familia Hydrochaeridae. Son roedores caviomorfos (Mesa \& Sánchez 2002), cuya distribución se da en América Central y del Sur, desde Panamá hasta el Norte de Argentina (Nowak 1991). En la actualidad son los roedores de mayor tamaño encontrados, con un peso que puede oscilar entre 35 y $65 \mathrm{~kg}$ (Emmons \& Feer 1997). Además, tienen hábitos semiacuáticos que habitan cerca de las orillas de los caños, lagunas o zonas anegadas, rodeadas de sabana, generalmente cerca de una franja de bosque.
Los capibaras son animales sociales, sedentarios y pacíficos (Ojasti 1980). Por lo general se encuentran en agrupaciones familiares compuestas por animales de ambos sexos, con predominancia de las hembras (Herrera 1999) en una relación de 3:1 (Alho et al. 1989), aunque la proporción de sexos al momento del nacimiento es de 53 machos por cada 47 hembras (Nogueira-Filho \& Nogueira 2004). $\mathrm{Su}$ sistema social muestra una jerarquía estrictamente dominada por los machos (GonzálezJiménez 1995), dentro de la cual el macho dominante es el tiene la mayoría de los apareamientos, aunque los subordinados obtienen 
un porcentaje significativo de éstos (Herrera \& Macdonald 1989). La dispersión post-natal de los capibaras ocurre al año de edad y se presenta de igual forma en ambos sexos (Herrera 1999). El tamaño y la estructura de los grupos oscila estacionalmente (Ojasti 1973, Herrera \& MacDonald 1989, Soini \& Soini 1992). Durante la época de sequía, la cohesión social es baja y los capibaras se agregan de forma temporal alrededor de los cuerpos de agua remanentes. Al parecer durante este tiempo hay reorganización de la estructura social de las manadas e intercambio de individuos entre ellas (Ojasti 1973). Al iniciar la época de lluvias, las agregaciones se separan y los grupos se dispersan.

Algunos autores designan a la población centroamericana y a la que se encuentra en la zona del océano Atlántico, en los valles de los ríos Magdalena, Cauca, Sinú y Atrato y en el Departamento de Chocó, todas ellas en Colombia, como una especie diferente (H. isthmius) (Goldman 1912), debido a su menor tamaño (Harrison-Matthews 1977), con un peso oscilando entre 26 y $28 \mathrm{~kg}$ y una gestación más corta (104-111 días) (Trapido 1949). También parece existir evidencia citogenética de que podría tratarse de dos especies diferentes (Peceño 1983). De hecho, Wilson \& Reeder (2005) en su catálogo sistemático reconocen ambas especies: sin embargo, no se han realizado estudios moleculares que muestren la diferenciación específica de esta población centroamericana y de la zona norte de Colombia.

La utilización de capibaras constituye una fuente de recursos económicos importante en las regiones donde habitan. Los humanos obtienen productos como carne (Alho et al. 1989; en sabanas inundables la producción de carne es 2.6 veces mayor a la del ganado vacuno), piel (Bolkovic et al. 2006) y grasa, en Venezuela, Brasil, Colombia, Perú, Ecuador y Argentina. Aunque no se encuentra reportada en CITES (Convención sobre el Comercio Internacional de Especies Amenazadas de Fauna y Flora Silvestres) ni en la UICN (Unión Internacional para la Conservación de la Naturaleza) dentro de alguna categoría de amenaza, la explotación de esta especie ha ocasionado la extinción local de algunas poblaciones (Ojasti 1991, Ballesteros \& Jorgenson 2009) o, al menos, la disminución del tamaño poblacional, como ocurre en el área objeto de este estudio (Aldana-Domínguez et al. 2007). Para mitigar esta situación se han planteado estrategias de manejo para recuperar las poblaciones de capibaras tales como la reintroducción, el reforzamiento poblacional y el enriquecimiento del hábitat. Sin embargo, para implementarlas es necesario conocer varios aspectos de la estructura genética de las poblaciones que den herramientas para el planteamiento de dichas estrategias de manejo. Aunque son muy escasos los estudios genéticos relacionados con las capibaras, si se han realizado múltiples estudios de esta especie desde otras perspectivas. Por ejemplo, manejo físico de la especie en cautiverio (Moreira 1993), comportamiento (González 2001, IssaRickli et al. 2008), infanticidio (Siqueira da Cunha Nogueira \& Nogueira-Filho 1997), cría (Álvarez et al. 1999), uso de hábitat (Arteaga 2001, Aldana-Domínguez et al. 2004, Maldonado-Chaparro et al. 2006, Atuesta-Dimian et al. 2006, Guzmán-Lenis et al. 2006), estimaciones poblacionales (Patiño et al. 2001, Prada \& Martínez 2001, Goncalves-Saraiva \& Da Silva-Rocha 2006, Aldana-Domínguez \& Ángel-Escobar 2007, Mulligan et al. 2007), determinación de áreas con valor de conservación para la especie (Pardo et al. 2006, Vieira-Muñoz 2007, Schivo et al. 2010, Acosta 2010), aprovechamiento comercial (Álvarez \& Kravetz 2004, Mesa-González et al. 2006, Pinheiro-Bartha et al. 2006, Lozada-Perdomo 2007) y estructura poblacional (CerqueiraLopes et al. 2006, Payán 2007, Ballesteros \& Jorgenson 2009).

El conocimiento de los parámetros genéticos de una población brinda elementos útiles para el planteamiento de estrategias de manejo de especies objeto de conservación, uso y aprovechamiento, y permite elegir los procedimientos adecuados para el mantenimiento de las poblaciones, como las traslocaciones de individuos (Sugg et al. 1996) o la cría en cautiverio. De acuerdo con Dobson \& Zinner (1998), las propiedades genéticas de una 
población restringen e influencian el potencial evolutivo de una especie, por lo que las poblaciones con mayor variabilidad genética tienen una mayor capacidad para reaccionar a los cambios ambientales.

Hasta el momento los estudios genéticos en capibaras han sido incipientes. Sin embargo, debido a su importancia dentro de los renglones económicos, a la situación de disminución de sus poblaciones en varios lugares donde son objeto de aprovechamiento y a su valor ecológico, se han adelantado algunos estudios sobre la variabilidad genética de las poblaciones, haciendo uso de ADN mitocondrial (Campos-Krauer 2009, Campos-Krauer \& Wisely 2010). Herrera et al. (2004) construyó una biblioteca genómica donde identificó 14 marcadores microsatelitales específicos para esta especie (en inglés, "short tandem repeat polymorphisms", STRPs). Estos marcadores están compuestos de unidades de repetición de dos a seis pares de bases (Weber \& Wong 1993) y están distribuidos al azar, encontrándose frecuentemente en los genomas eucarióticos. Al presentar elevados niveles de variabilidad genética son una herramienta de gran utilidad para la diferenciación de poblaciones, la evaluación de la variabilidad genética de una población y los estudios de paternidad y parentesco de individuos en una población (Paetkau et al. 1995, McConnell et al. 1997). La pequeña cantidad de ADN necesaria para el análisis de estos marcadores moleculares ha permitido a los investigadores utilizar procedimientos no invasivos durante el muestreo de animales silvestres lo cual permite la obtención de importante conocimiento de la dinámica poblacional de esas especies (Bruford \& Wayne 1993).

Los objetivos principales del presente estudio son los siguientes: 1- Determinar el nivel de diversidad genética en una población de capibaras de Hato Corozal en el Departamento de Casanare (Colombia) mediante cinco marcadores microsatelitales; 2- Determinar la posible existencia de equilibrio Hardy-Weinberg en esa población para esos marcadores; 3- Determinar posibles diferencias en las frecuencias génicas de machos y hembras de esa población;
4- Determinar si la población históricamente ha permanecido con tamaño constante o ha podido sufrir fenómenos de cuello de botella o de expansión poblacional.

\section{MATERIAL Y MÉTODOS}

Muestras: En octubre de 2007 se capturaron 31 ejemplares de capibaras en predios del Hato Andalucía, en el Municipio de Hato Corozal, Departamento de Casanare, Colombia $\left(06^{\circ} 08^{\prime} 58^{\prime \prime} \mathrm{N}-71^{\circ} 48^{\prime} 26^{\prime \prime} \mathrm{W}\right)$. Estos animales representaron más del $50 \%$ de la manada presente en el Hato citado. A los 31 individuos capturados se les tomó una muestra de sangre de la vena femoral, las cuales fueron depositadas en tarjetas FTA $^{\circledR}$ (Whatman Bioscience, Cambridge, United Kingdom, 2003; temperatura ambiente $23^{\circ} \mathrm{C}$ ) y preservadas a temperatura ambiente hasta su procesamiento en el laboratorio. Los animales fueron capturados siguiendo los protocolos aprobados por el comité de ética de la Universidad Javeriana y las leyes del Ministerio de Ambiente, Vivienda 170 y Desarrollo Territorial (R. 1252) de Colombia.

Análisis molecular: El ADN genómico de cada individuo fue extraído siguiendo el procedimiento recomendado por la casa comercial Whatman FTA ${ }^{\circledR}$ protocols (2003); se analizaron cinco microsatélites seleccionados de una lista de 14 microsatélites desarrollados para $H$. hydrochoerus por Herrera et al. (2004), de acuerdo con su grado de variabilidad (Cuadro 1).

Una vez extraído el ADN se amplificó cada locus mediante la técnica del PCR (Polimerase Chain Reaction) en las 31 muestras recolectadas, de acuerdo con las condiciones de PCR descritas por Herrera et al. (2004). Así, se utilizó, aproximadamente, 20ng de ADN en un volumen total de reacción de $25 \mu \mathrm{L}$, con $0.3 \mu \mathrm{M}$ de cada cebador (primer), $0.3 \mathrm{mM}$ dNTP, $1 \mathrm{x}$ Taq buffer $\left(1.5 \mathrm{mM} \mathrm{MgCl}_{2}, 10 \mathrm{mM}\right.$ Tris- $\mathrm{HCl}$, $50 \mathrm{mM} \mathrm{KCl}$ ) y 0.75 unidades de Taq polimerasa. Los cebadores utilizados fueron Capy 12, Capy 14, Capy 24, Capy 25, Capy 26 (Herrera et al. 2004).

La amplificación se realizó en un termociclador PTC-100 25612 (MJR Research, Inc), iniciando con una desnaturalización de 
CUADRO 1

Caracterización de los cinco marcadores microsatelitales aplicados al grupo muestreado de capibaras en los Llanos Orientales de Colombia

TABLE 1

Characteristics of the five microsatellite markers applied to one troop of capybaras sampled in the Colombian Eastern Llanos

\begin{tabular}{|c|c|c|c|}
\hline Locus & Secuencia del primer $\left(5^{\prime}-3^{\prime}\right)$ & $\#$ de alelos & Rango de tamaño de alelos (pb) \\
\hline Capy 12 & $\begin{array}{l}\text { TGGGTGCCAGAATGCATAGTC } \\
\text { GCATTGCCACCCCTACCTTA }\end{array}$ & 2 & $195-197$ \\
\hline Capy 14 & $\begin{array}{l}\text { TCATAAAGCTGTCCCACTCTGC } \\
\text { GCTGGAAAGCATAGCTCAAACG }\end{array}$ & 2 & $285-287$ \\
\hline Capy 24 & $\begin{array}{l}\text { TGCAGGGAGCACTTTATCCA } \\
\text { CAAGCTGGGCACAAAAAGGA }\end{array}$ & 7 & $145-157$ \\
\hline Capy 25 & $\begin{array}{l}\text { CAAGCATGTTCCTTTCCCCTTA } \\
\text { TGGAGAGCAGAGGATTAAAAGCA }\end{array}$ & 3 & $260-264$ \\
\hline Capy 26 & $\begin{array}{l}\text { TCTGCTTGGTCCTTCTGTTTGA } \\
\text { TCGCAAGGGGACTCTAGTGTTA }\end{array}$ & 2 & $274-276$ \\
\hline
\end{tabular}

Las características de esos cinco microsatélites fueron determinadas por Herrera et al. 2004.

The characteristics of these five microsatellites were determined by Herrera et al. 2004.

$95^{\circ} \mathrm{C}$ durante 5 minutos, seguida de 34 ciclos de 30 segundos de desnaturalización a $94^{\circ} \mathrm{C}$, 30 segundos a la temperatura óptima de anillamiento $\left(54,58,60\right.$ y $61^{\circ} \mathrm{C}$ dependiendo del marcador) y 45 segundos de extensión a $72^{\circ} \mathrm{C}$. Finalmente, una fase de extensión de 5 minutos a $72^{\circ} \mathrm{C}$. Los productos de PCR se analizaron por medio de electroforesis en gel de poliacrilamida al $12 \%$ en una cámara vertical de Secuencia ADN Thermo EC EC-160. La electroforesis se prolongó por unas 2-6 horas, dependiendo del tamaño del marcador, a $15 \mathrm{~W}$ constantes y a un voltaje que osciló entre los 300-400 V. La muestra sometida a electroforesis consistió en una mezcla de $2 \mu \mathrm{L}$ de producto de amplificación y $3 \mu \mathrm{L}$ de tampón de carga (azul de bromofenol $2 \% \mathrm{p} / \mathrm{v}$, disuelto en agua MiliQ). Posteriormente, los marcadores fueron visualizados por exposición del gel a luz blanca, previa tinción con nitrato de plata $\left(\mathrm{AgNO}_{3}\right)$. El tamaño de los productos amplificados fue estimado utilizando un estándar de $10 \mathrm{pb}$ y $50 \mathrm{pb}$ (Invitrogen). Los genotipos y tamaños alélicos fueron registrados por conteo directo.

La diversidad genética de la población de $H$. hydrochaeris analizada fue estimada mediante diversos estadísticos: número de alelos por locus $(\mathrm{N})$, heterocigocis esperada $\left(\mathrm{H}_{\mathrm{e}}\right)$ y heterocigosis observada $\left(\mathrm{H}_{\mathrm{o}}\right)$, además del contenido de información polimórfica (PIC, Botstein et al. 1980). Para calcular esos estadísticos se utilizó el software Cervus versión 3.03 (Marshall et al. 1998).

A través del estadístico $G$ se evaluaron las desviaciones del desequilibrio de ligamiento para probar la hipótesis de independencia de genotipos entre los loci. Para ello se empleó el programa GENEPOP 4.0 (Raymond \& Rousset 1995).

El posible desequilibrio Hardy-Weinberg $(\mathrm{H}-\mathrm{W})$ se analizó mediante tres métodos diferentes (con el programa GENEPOP 4.0, Raymond \& Rousset 1995): la F de Weir \& Cockerham (1984), la f de Robertson \& Hill (1984) y mediante probabilidades exactas con el procedimiento de Cadenas de Markov Monte Carlo (MCMC) (Raymond \& Rousset 1995), con 1000 demorizaciones, 50 "batches" y 1000 iteraciones por "batch".

Adicionalmente, se realizó un test global para evaluar si la población muestra una desviación del equilibrio $\mathrm{H}-\mathrm{W}$, usando el 
método de Fisher (Raymond \& Rousset 1995), con todos los microsatélites analizados tomados simultáneamente.

Para evaluar la heterogeneidad genética entre los machos y las hembras del grupo capturado se empleó un test exacto con el método de Fisher con 1000 demorizaciones, 50 "batches" y 1000 iteraciones por "batch" utilizando las frecuencias genotípicas encontradas en los machos y hembras (14 machos y 17 hembras).

Para determinar si la población de capibaras estudiada ha sufrido un cuello de botella reciente se utilizó el programa Bottleneck (Cornuet \& Luikart 1996, Piry et al. 1999). Aquellas poblaciones que han experimentado cuellos de botella recientes tienen el número de alelos y los niveles de heterocigosis esperada inferiores a los de poblaciones que no han experimentado ese fenómeno. Sin embargo, el número de alelos se reduce más rápidamente que la heterocigosis esperada. Por lo tanto, la heterocigosis esperada calculada por métodos de coalescencia a partir del número de alelos es inferior a la heterocigosis esperada a partir de las frecuencias alélicas observadas. Para calcular la probabilidad de un cuello de botella reciente en la población de capibaras estudiada se utilizaron cuatro procedimientos: prueba del signo, prueba de las diferencias estandarizadas, prueba de Wilcoxon y el método gráfico de Luikart et al. 1998. Además, el programa Bottleneck permite utilizar las opciones "Two Phases Model" (TPM, Di Rienzo et al. 1994) y "Stepwise Mutation Model" (SMM, Ohta \& Kimura 1973), que describen adecuadamente los modelos mutacionales de los marcadores microsatelitales.

Otros procedimientos utilizados para determinar posibles cambios demográficos en la población de capibaras estudiada fueron: 1La prueba de Kimmel et al. (1998); se define el índice de desbalance $\beta$ como:

$\beta(\mathrm{t})=\mathrm{E}\left(\theta_{\mathrm{v}}\right) / \mathrm{E}\left(\theta_{\mathrm{Po}_{\mathrm{o}}}\right)=\mathrm{V}(\mathrm{t}) /\left[\left(\left(1 / \mathrm{P}_{\mathrm{o}}{ }^{2}\right)-1\right) / 2\right.$, o también, $\ln \beta(\mathrm{t})=\ln \theta_{\mathrm{v}}-\ln \theta_{\mathrm{Po}}=\ln (\mathrm{V})-\ln$ $\left(\left(\left(1 / \mathrm{P}_{\mathrm{o}}{ }^{2}\right)-1\right) / 2\right)$, donde $\theta_{\mathrm{v}}$ es la varianza de los tamaños de los tándem de repetición y $\theta_{\mathrm{Po}}$ $=\left(1 /\left(\mathrm{P}_{\mathrm{o}}^{2}-1\right)\right) / 2$, con $\mathrm{P}_{\mathrm{o}}$ siendo un estimador de homocigosis.
Si una población está en equilibrio, tiene un tamaño demográfico constante, y no ha sufrido una expansión poblacional, $\beta=1$ ( $\ln$ $\beta=0$ ). Por el contrario, si una población ha experimentado una expansión proviniendo de una situación de equilibrio mutación-deriva (tamaño poblacional constante), $\beta<1 \quad(\ln \beta<0)$. Si una población ha experimentado una expansión viniendo previamente de un cuello de botella, $\beta>1(\ln \beta>0)$. Este último valor se presentará por un largo tiempo (unas cuantas miles de generaciones) antes de mostrar la firma de una expansión poblacional $(\beta<1(\ln \beta<0))$. Hay una excepción a esta regla general, cuando el cuello de botella es tan intenso que la población se convierte en monomórfica antes de la expansión demográfica, en cuyo caso, $\beta<1 \quad(\ln \beta<0)$ todas las veces. Todos los valores $\beta$ son consistentes con modelos de crecimiento poblacional "stepwise", logístico o exponencial y no están específicamente afectados por diferentes modelos mutacionales (Kimmel et al. 1998).

Dos métodos fueron empleados para determinar la significación estadística de $\beta(\ln \beta)$ obtenida en la población de capibaras. La primera fue la aplicación del procedimiento "jackknife" (Efron \& Tibshirani 1993) para obtener la varianza de $\beta$ y, con esta varianza, se estimaron un test de Student y un intervalo de confianza del 95 y del $99 \%$. Un segundo procedimiento utilizado fue mediante las distribuciones empíricas de $\ln \beta$ a partir de 500 simulaciones de coalescencia con un $\theta=5$. En este caso, el intervalo de confianza al $95 \%$ fue determinado como $(-0.23,0.25)$.

Un segundo test empleado fue el de Zhivotovsky et al. (2000), el cual calcula un índice de expansión:

$$
\mathrm{S}_{\mathrm{k}}=1-\left(\left(\mathrm{K}-\left(\mathrm{R}_{\mathrm{k}} \mathrm{V} / 2\right) / 5 \mathrm{~V}^{2}\right)\right.
$$

dónde $\mathrm{K}$ y $\mathrm{V}$ son la curtosis no normalizada (cuarto momento central) y la varianza del tamaño alélico, mientras $\mathrm{R}_{\mathrm{k}}=\mathrm{k}_{\mathrm{m}} / \sigma_{\mathrm{m}}^{2}$ (son la curtosis y la varianza en el número de cambios mutacionales en las repeticiones). El valor de $\mathrm{R}_{\mathrm{k}}$ empleado fue 6.3 , debido a que este valor fue obtenido para microsatélites dinucleótidos 
por Dib et al. (1996), y siendo los microsatelites empleados en este estudio precisamente dinucleótidos. El valor de $S_{k}$ es esperado que sea igual a cero en un modelo mutacional "stepwise" general y simétrico para una población en equilibrio y de tamaño constante (esto fue derivado por Zhivotovsky \& Feldman 1995). El estadístico $S_{k}$ es positivo si una expansión afectó a la población y es negativo si un cuello de botella afectó a la población. Para obtener conclusiones demográficas de este análisis, la varianza intra-poblacional y el índice de expansión son promediados para todos los microsatellites estudiados. Para medir la significación estadística de los valores de $\mathrm{S}_{\mathrm{k}}$, dos métodos fueron empleados. Primero, se llevó a cabo un "bootstrap" para los loci (10 000 corridas). En segundo lugar, como en el análisis anterior, se utilizó un procedimiento "jackknife" para obtener la varianza de $S_{k} y$, con esta varianza, un test $\mathrm{t}$ de Student y el intervalo de confianza al $95 \%$ fueron estimados.

El último análisis demográfico aplicado a la población estudiada de capibaras fueron el test de curtosis intra-locus (k) y el test interlocus (g) propuesto por Reich \& Goldstein (1998) y Reich et al. (1999). El primer test mide diferencias entre distribuciones alélicas con picos múltiples de poblaciones de tamaño constante y distribuciones alélicas de un único pico más suave típicas de una población en expansión:

$$
\mathrm{k}=2.5 \mathrm{Sig}^{4}+0.28 \operatorname{Var}-(0.95 / \mathrm{n})-\mathrm{Gam}_{4},
$$

donde $\mathrm{Sig}^{4}$ es la varianza insesgada al cuadrado del tamaño alélico, Var es la varianza de muestreo del mismo concepto y $\mathrm{Gam}_{4}$ es el momento central cuarto insesgado del alelo, respectivamente. Para determinar los niveles de significación, se utilizó una distribución binomial con el número de ensayos igual al número de loci basado en lo esperado en al menos igual probabilidad de valores de $\mathrm{k}$ positivos $\mathrm{y}$ negativos para el conjunto de loci analizados $(\mathrm{p}=0.515)$.

El test $g$ se basa en los siguientes hechos. Este test inter-locus muestra que para poblaciones estables, la varianza es altamente variable entre diferentes loci analizados, mientras en poblaciones en expansión esta varianza es usualmente baja. Así pues, tamaños alélicos con varianzas suficientemente bajas pueden tomarse como evidencia para expansiones poblacionales. El test usado para diferencias estadísticas es:

$$
\mathrm{g}=\frac{\operatorname{Var}\left[V_{j}\right]}{4 / 3 \bar{V} 2+1 / 6 \bar{V}}
$$

donde $\operatorname{Var}\left(\mathrm{V}_{\mathrm{j}}\right)$ es la varianza observada de las varianzas de los tamaños alélicos a partir de los marcadores empleados, y V es la varianza promedio de todos los loci. Un bajo valor de g se toma como un signo de expansión poblacional. Los niveles de significación para este test interlocus se encuentran en la Tabla 1 de Reich et al. (1999), la cual muestra los cortes del percentil del 5\% para este test. Para el caso presente, los valores de g menores a 0.08 indicarán una expansión poblacional. Luikart et al. (1998) afirmaron que este último test es probablemente el más poderoso para esta función.

\section{RESULTADOS}

Diversidad genética: Los cinco loci microsatélitales empleados resultaron ser polimórficos (Cuadro 2). Esos loci mostraron entre 4 y 9 alelos y una heterocigosis esperada $\left(\mathrm{H}_{\mathrm{e}}\right)$ entre 0.41 (Capy12) y 0.87 (Capy24). $\mathrm{La} \mathrm{H}_{\mathrm{e}}$ promedio para los cinco loci fue de $0.606 \pm 0.174$ mientras que el número de alelos promedio por locus fue de $5.20 \pm 2.17$.

\section{Equilibrio H-W y desequilibrio de liga-} miento: De los cinco loci analizados, tres no se desviaron significativamente de las proporciones genotípicas esperadas en equilibrio H-W (Capy24, 25, 26) (Cuadro 3). Capy12 presentó un significativo exceso de homocigotos $(\mathrm{p}=0.0000 \pm 0.0000)$, mientras que Capy14 presentó un significativo exceso de heterocigotos $(\mathrm{p}=0.01 \pm 0.01)$. Al tomar simultáneamente los cinco loci, se detectó un significativo exceso de homocigotos al aplicar el método 
CUADRO 2

Estadísticos de diversidad genética determinados en cinco loci microsatelitales en una población de capibaras

TABLE 2

Gene diversity statistics determined in five microsatellite loci in a capybara population

\begin{tabular}{ccccc} 
locus & No. Alelos & $\mathrm{CIP}$ & $\mathrm{H}_{\mathrm{o}}$ & $\mathrm{H}_{\mathrm{e}}$ \\
Capy12 & 4 & 0.35 & 0.063 & 0.41 \\
Capy14 & 4 & 0.42 & 0.81 & 0.54 \\
Capy24 & 9 & 0.84 & 0.77 & 0.87 \\
Capy25 & 5 & 0.6 & 0.52 & 0.67 \\
Capy26 & 4 & 0.42 & 0.42 & 0.54 \\
\hline
\end{tabular}

Se indican el número de alelos (No. Alelos), la heterocigosis observada $\left(\mathrm{H}_{\mathrm{o}}\right)$, la heterocigosis esperada $\left(\mathrm{H}_{\mathrm{e}}\right)$ y el contenido de información polimórfica (CIP).

There is noted the number of alleles (No. Alleles), the observed heterozygosity $\left(\mathrm{H}_{\mathrm{o}}\right)$, the expected heterozygosity $\left(\mathrm{H}_{\mathrm{e}}\right)$ and the polymorphic information content (CIP).

de Fisher $(\chi=53.48$, g.l $=10, p=0.0000)$. No se dio ningún caso de desequilibrio de ligamiento entre ningún par de loci. Esto significa que la información evolutiva que aporta cada uno de los cinco loci microsatelitales es independiente para cada uno de ellos.

\section{Diferenciación genotípica entre machos} y hembras en la manada capturada: El análisis de diferenciación genotípica mostró que las frecuencias genotípicas no difieren significativamente entre los machos y las hembras de la manada capturada. Por lo tanto, los machos y hembras parecen provenir de un mismo acervo genético $\left(\chi^{2}=7.56, \mathrm{~g} . \mathrm{l}=10, \mathrm{p}=0.672\right)$.

Cambios demográficos históricos: $\mathrm{El}$ análisis de Cornuet \& Luikart (1996) mostró que, independientemente del modelo mutacional elegido, los cinco loci microsatelitales presentaron valores de heterocigosis esperada superiores a los valores de heterocigosis esperada estimados a partir del número de alelos encontrados. Para cuatro de los cinco marcadores esas diferencias fueron estadísticamente significativas. Esto sugiere que la población ha pasado recientemente por un cuello de botella.
Tanto el test de las diferencias estandarizadas y el test del signo (TPM, $\mathrm{p}=0.032$, SMM, $\mathrm{p}=0.040$ ) como el test de Wilcoxon (TPM, $\mathrm{p}=0.031$, SMM, $\mathrm{p}=0.031$ ), al igual que el descriptor gráfico de Luikart et al. (1998) ratificaron el posible cuello de botella reciente en esta población de capibaras de los Llanos orientales colombianos. Los restantes análisis demográficos básicamente ratificaron esta situación. El test de Kimmel et al. (1998) mostró un valor de $\beta=0.8476$ (ln $\beta=-0.1653)$. Esos valores son significativamente menores que $\beta=1(\ln \beta=0)$, cuyo intervalo de confianza para $\beta$ es 1.83-3.05. Cuando los valores de $\beta$ y $\ln \beta$ son significativamente superiores a uno y cero, respectivamente, es una indicación de que la población analizada pasó por un cuello de botella y, posteriormente, experimentó una expansión poblacional. Por el contrario, cuando $\beta$ y $\ln \beta$ son menores que uno y cero, respectivamente, cómo es el caso, es síntoma de una población que ha atravesado un cuello de botella muy intenso sin posterior expansión poblacional. Luego, la población analizada de capibaras parece haber sufrido un fuerte cuello de botella sin posterior expansión poblacional. El test de Zhivotovsky et al. (2000) no mostró un cuello de botella tan claro como los procedimientos previos. El valor de $S_{k}$ fue 0.6059 , lo cual no difirió significativamente de $\mathrm{S}_{\mathrm{k}}=0$ (intervalo de confianza del 99\%, -0.60, 0.74), lo que indicaría una población de tamaño histórico constante. Sin embargo, los valores promedios de $\mathrm{k}$ y de la varianza fueron mucho menores que los obtenidos en otros estudios ( $\mathrm{k}=4.9699$ y Var $=0.9787$, respectivamente), característica clara de una población con poca variabilidad genética probablemente perdida por un cuello de botella. Los tests $\mathrm{k} \mathrm{y} \mathrm{g}$, diseñados para detectar expansiones poblacionales, resultaron claramente negativos. La probabilidad del test $\mathrm{k}$ fue uno, dato que indica totalmente lo contrario de una expansión poblacional, esto es, un cuello de botella. Lo mismo sucede con el test g: para cinco loci y 31 muestras analizadas el valor crítico de g por debajo del cual se detectaría una expansión poblacional es 0.08 . Por el contrario, se obtuvo un valor de $\mathrm{g}=1.4253$, 
CUADRO 3

Frecuencias alélicas de los cinco loci microsatelitales utilizados

TABLE 3

Allele frequencies of the five microsatellite loci employed

\begin{tabular}{|c|c|c|c|c|c|c|c|}
\hline \multirow{2}{*}{ locus } & \multirow{2}{*}{ Tamaño Alelo (pb) } & \multirow{2}{*}{$\mathrm{N}$} & \multirow{2}{*}{ Frecuencia } & \multicolumn{2}{|c|}{$F_{j s}$} & \multirow{2}{*}{$p$} & \multirow{2}{*}{ S.E } \\
\hline & & & & $\mathrm{W} \& \mathrm{C}$ & $\mathrm{R} \& \mathrm{H}$ & & \\
\hline \multirow[t]{3}{*}{ Capy 12} & 95 & 15 & 0.24 & 0.91 & & $0^{*}$ & - \\
\hline & 97 & 47 & 0.76 & 0.91 & & & \\
\hline & Tot & 62 & & 0.91 & 0.94 & & \\
\hline \multirow[t]{3}{*}{ Capy14 } & 85 & 30 & 0.50 & -0.59 & & $0.01 *$ & - \\
\hline & 87 & 30 & 0.50 & -0.59 & & & \\
\hline & Tot & 60 & & -0.59 & -0.59 & & \\
\hline \multirow[t]{8}{*}{ Capy24 } & 45 & 6 & 0.10 & 0.28 & & 0.15 & 0.01 \\
\hline & 47 & 6 & 0.10 & -0.09 & & & \\
\hline & 49 & 13 & 0.22 & 0.13 & & & \\
\hline & 51 & 9 & 0.15 & 0.10 & & & \\
\hline & 53 & 9 & 0.15 & -0.16 & & & \\
\hline & 55 & 8 & 0.13 & 0.15 & & & \\
\hline & 57 & 9 & 0.15 & 0.10 & & & \\
\hline & Tot & 60 & & 0.07 & 0.07 & & \\
\hline \multirow[t]{4}{*}{ Capy25 } & 60 & 14 & 0.25 & -0.13 & & 0.09 & - \\
\hline & 62 & 15 & 0.27 & 0.38 & & & \\
\hline & 64 & 27 & 0.48 & 0.37 & & & \\
\hline & Tot & 56 & & 0.23 & 0.19 & & \\
\hline \multirow[t]{3}{*}{ Capy26 } & 74 & 30 & 0.50 & 0.22 & & 0.2 & - \\
\hline & 76 & 30 & 0.50 & 0.22 & & & \\
\hline & Tot & 60 & & 0.22 & 0.22 & & \\
\hline
\end{tabular}

Se indica el número de alelos analizados $(\mathrm{N})$, el coeficiente de endogamia $\left(\mathrm{F}_{\mathrm{is}}\right)$ medido con el método de Weir \& Cockerham (1984) (W\&C), con el método de Robertson \& Hill (1984) (R\&H) y con probabilidades exactas (P) para la población de capibaras analizada. S.E.=Desviación Estándar, Tot=Total, pb=pares de bases, $*=$ probabilidades significativas.

It is noted the number of alleles analyzed $(\mathrm{N})$, the endogamy coefficient $\left(\mathrm{F}_{\text {is }}\right)$ measured with the Weir \& Cockerham (1984) (W\&C)'s method, the Robertson \& Hill (1984) (R\&H)'s method and exact probabilities (P) for the capybara population analyzed. $\mathrm{S} . \mathrm{E} .=$ Standard deviation, Tot=Total, $\mathrm{Pb}=$ base pairs, ${ }^{*}=$ significant probabilities.

que es extremadamente elevado. Esto denotó lo contrario de una expansión poblacional. De nuevo, pues, este test mostró fuerte evidencia de un cuello de botella.

\section{DISCUSIÓN}

En el presente estudio presentamos el primer análisis genético molecular de una población de $H$. hycrochoerus que habita en los Llanos orientales de Colombia aplicando cinco marcadores microsatelitales. H. hycrochoerus puede constituirse en una especie supremamente interesante para estudios que analicen la variabilidad genética intrapoblacional y cómo la estructura social puede repercutir en la estructura genética de una población (Chesser 1991a, 1991b, Chesser et al. 1993, Pope 1992, Ruiz-García 2010a), debido a sus hábitos poligínicos (Ojasti 1973), a su plasticidad en la respuesta social (modificación del tamaño de grupos) de acuerdo a las condiciones climáticas 
(Ojasti 1991, Maldonado-Chaparro \& Sánchez 2010) y a la relativa facilidad para poder capturar los ejemplares de manadas enteras debido a su fuerte cohesividad grupal y a sus hábitos ecológicos.

El número promedio de alelos encontrado en la población de capibaras puede considerarse moderadamente elevado (5.2 alelos). Por ejemplo, Dallas et al. (1995) encontraron un promedio de cinco alelos para un sistema de seis loci en una población de ratón doméstico (Mus musculus). Sin embargo, la $\mathrm{H}_{\mathrm{e}}$ refleja mejor la diversidad genética que el número promedio de alelos porque es independiente del tamaño muestral (Nei et al. 1975). El nivel promedio de $\mathrm{H}_{\mathrm{e}}$ para el grupo estudiado de capibaras $\left(\mathrm{H}_{\mathrm{e}}=0.61\right)$ puede considerarse medio para este tipo de marcadores. Es superior al que se encuentra en algunas especies de la fauna neotropical como el oso andino (Tremarctos ornatos; $\mathrm{H}_{\mathrm{e}}=0.45-0.55$; Ruiz-García 2010c, Ruiz-García et al. 2005), el gato andino (Leopardus jacobita; $\mathrm{H}_{\mathrm{e}}=0.52$; Cossios et al. 2010) $\mathrm{o}$ el delfín rosado (Inia geoffrensis; $\mathrm{H}_{\mathrm{e}}=0.53$; Ruiz-García 2010b), inferior al nivel promedio de $\mathrm{H}_{\mathrm{e}}$ encontrado en otras especies de la fauna neotropical, como, por ejemplo, el ocelote $(L$. pardalis; $\mathrm{H}_{\mathrm{e}}=0.92$; Ruiz-García 2001), el margay (L. wiedii; $\mathrm{H}_{\mathrm{e}}=0.85$; Ruiz-García 2001), el jaguar (Panthera onca; $\mathrm{H}_{\mathrm{e}}=0.85$; Ruiz-García et al. 2006) o Peromyscus melanosis $\left(\mathrm{H}_{\mathrm{e}}=0.8\right.$ 0.95; Chirhart et al. 2005), pero similar al encontrado en especies neotropicales como el venado rojo y el venado coliblanco (Mazama americana y Odocoileus virginianus; $\mathrm{H}_{\mathrm{e}}=0.64$ y 0.61, respectivamente; Ruiz-García et al. 2009) u otro roedor como Oryzomys couesi $\left(\mathrm{H}_{\mathrm{e}}=0.68\right.$; Vega 2006). En cuanto al contenido de información polimórfica (PIC) solo los marcadores Capy24 y 25 pueden ser considerados muy informativos, según la clasificación de Botstein et al. (1980), en la cual marcadores con valores de PIC superiores a 0.5 son considerados muy informativos, valores entre $0.25 \mathrm{y}$ 0.50 mediamente informativos y valores inferiores a 0.25 , son poco informativos.

De acuerdo con Chiappero et al. (2002), niveles elevados de polimorfismo pueden ser mantenidos en poblaciones naturales a través de mecanismos como el número efectivo de la población, dado que un aumento en el tamaño poblacional reduce el efecto de la deriva genética. En especies sociales, como la capibara, con variaciones estacionales del tamaño poblacional (Maldonado-Chaparro \& Sánchez 2010), estos mecanismos pueden ser la fuente de la variabilidad genética al interior de los grupos. El establecimiento de nuevos grupos de cría cada año con individuos no relacionados puede dispersar la variabilidad genética entre los grupos en una escala microgeográfica (Ruiz-García 1991, 1993, 1998, Storz, 1999). Los niveles medios de variabilidad genética encontrados en esta población de capibaras pueden responder a esos fenómenos.

Tres de los cinco marcadores microsatelitales empleados mostraron no diferir significativamente del equilibrio $\mathrm{H}-\mathrm{W}$. En principio esto podría mostrar que los apareamientos al interior del grupo fueron aleatorios (al menos, respecto a los marcadores considerados) o que, si hay animales que se han integrados recientemente a ese grupo, éstos provienen de otras poblaciones que poseen el mismo acervo genético con respecto a los individuos del grupo analizado (Cockerham 1973). Sin embargo, dos loci mostraron una desviación significativa con respecto al equilibrio $\mathrm{H}-\mathrm{W}$ (Capy12 y Capy14). Capy12 mostró un enorme exceso de homocigotos. El exceso de homocigotos en una población podría ser el resultado de eventos de endogamia dentro de la misma (Allendorf \& Luikart 2007). Sin embargo, la endogamia afecta por igual a todo el genoma por lo que se esperaría que si este fenómeno fuera el más trascendente, los cinco marcadores empleados deberían mostrar un exceso de homocigotos, cosa que no ocurre en este caso. Otra posibilidad es la existencia de posible estructura genética por subdivisión, referido como efecto Wahlund. Si así fuera, eso significaría que existen diferencias marcadas entre las poblaciones cercanas de capibaras para ese marcador, pero no para los otros marcadores. $\mathrm{Si}$ esas diferencias para ese marcador no se han eliminado es porque el flujo génico entre 
poblaciones cercanas es limitado (aspecto que no muestran los otros marcadores) o porque Capy 12 está ligado algún gen sometido a selección natural que actúe diferencialmente a nivel micro o macro-espacial. Otra posibilidad es la presencia de alelos nulos en este locus (Callen et al. 1993), hecho presente en buena parte de estudios con microsatélites (Dakin \& Avise 2004). Los alelos nulos pueden resultar de problemas técnicos durante la PCR o debido a la alta tasa mutacional de este tipo de marcadores, que ocasiona una alteración de la secuencia entre poblaciones ligeramente distantes (Callen et al. 1993).

Por el contrario, el locus Capy14 presenta un significativo exceso de heterocigotos. Este fenómeno puede darse básicamente por dos fenómenos. El primero de ellos, es que existiera flujo génico diferencial para ambos sexos y que los machos y hembras inmigrantes que se incorporan reproductivamente en una nueva población, se reproduzcan rápidamente con los individuos residentes. Esto produciría un exceso de heterocigotos (Chesser 1991a, 1991b, Chesser et al. 1993, Ruiz-García 2010a) porque machos y hembras tendrían genotipos pertenecientes a acervos genéticos altamente diferenciados. Sin embargo, esta posibilidad entra en conflicto con algunas de las posibilidades explicitadas para el marcador anterior. La segunda posibilidad es que este marcador se encuentre ligado a algún otro gen que esté afectado por heterosis (selección positiva a favor de los heterocigotos) y que arrastre a Capy14 a mostrar un exceso de heterocigotos.

Sin embargo, el número de marcadores utilizados es muy pequeño para poder comprender que fuerzas evolutivas están modulando completamente el genoma de los capibaras de la población analizada. Es imprescindible, aumentar el número de marcadores microsatelitales para determinar si estas unidades poblacionales muestran un sistema reproductivo cerrado pero con cruzamientos aleatorios en el seno de la población para los marcadores analizados o si, por el contrario, existe un intercambio de genes ligados a la existencia de filopatría y migración diferencial según los sexos y si las poblaciones aledañas poseen, o no, acervos genéticos parecidos. Sin embargo, la no existencia de una diferencia entre los genotipos de machos y hembras, está en concordancia con el hecho de que en esta especie ambos sexos se dispersan (Ojasti 1991).

El análisis de cuello de botella con el método de Cornuet \& Luikart (1996) indicó que la población estudiada ha experimentado reducción en su tamaño efectivo poblacional $\left(\mathrm{N}_{\mathrm{e}}\right)$ en el pasado reciente. Reciente es un término relativo ya que puede significar entre $2 \mathrm{~N}_{\mathrm{e}}$ y $4 \mathrm{~N}_{\mathrm{e}}$ generaciones. Simplemente, si imagináramos un número efectivo de 100 animales, eso podría suponer entre 200 y 400 generaciones atrás. Eso significa que, en épocas recientes, la variabilidad genética de esta población o de la población que dio lugar al nucleo de capibaras estudiado se vio afectada de forma negativa. Los otros procedimientos revelaron resultados similares, especialmente las pruebas de Kimmel et al. (1998), k y g. Por ejemplo, el test de Kimmel et al. (1998) aplicado a otras especies neotropicales como los delfines rosados (Inia geoffrensis e Inia boliviensis) mostraron resultados muy diferentes a los aquí reportados para las capibaras. Tanto el delfín rosado de la Amazonía occidental $(\beta=2.1272$ y $\ln \beta=0.7548)$, como el delfín rosado boliviano $(\beta=2.1169$ y $\ln \beta=0.7499)$, mostraron haber pasado originalmente por un cuello de botella pero, posteriormente, haber sufrido una fuerte expansión poblacional (Ruiz-García 2010b). Contrariamente, la población estudiada de capibaras únicamente mostró haber atravesado un muy fuerte cuello de botella pero sin una posterior expansión poblacional. Esto denota que el cuello de botella ha sido muy reciente y que no ha transcurrido suficiente tiempo, o no se han dado las condiciones, para una posterior expansión poblacional. El único test que no reveló claramente este fuerte cuello de botella fue el de Zhivotovsky et al. (2000). Por ejemplo, el delfín rosado boliviano mostró haber atravesado un fuerte cuello de botella histórico con este procedimiento $\left(\mathrm{S}_{\mathrm{k}}=-2.2533\right)$, no así el delfín rosado de la Amazonía occidental $\left(\mathrm{S}_{\mathrm{k}}=0.2027\right)$. Aunque la población analizada de capibaras 
no mostró un valor de $S_{k}$ significativamente diferente de cero (población de tamaño constante), los valores promedios de $\mathrm{k}$ y Var fueron más pequeños que los encontrados para esas dos poblaciones de delfines rosados (Bolivia: $\mathrm{k}=19.2440$, Var=0.9951; Amazonía occidental: $\mathrm{k}=35.5744$, Var=2.6250), lo que aboga por una baja variabilidad genética en esa población.

Esta pérdida de la variabilidad genética puede reducir el potencial evolutivo de las poblaciones de capibaras en los Llanos Orientales colombianos y hacerla altamente vulnerable, por ejemplo, a nuevas enfermedades. Parece ser que existe múltiple evidencia de poblaciones de capibaras en decrecimiento en los Llanos orientales de Colombia. Ya Hernández-Camacho et al. (1983) habían detectado una drástica disminución de las poblaciones de capibaras en los departamentos de Arauca y Casanare. ICN (2002) indicó que las poblaciones de capibaras en el Departamento de Vichada están disminuyendo; en el Departamento de Arauca, las poblaciones de capibaras han sido diezmadas por el tráfico de carne seca hacia Venezuela (Aldana-Domínguez et al. 2002). La demanda de carne seca a Venezuela asciende entre 120000 a 150000 individuos por año procedentes del comercio ilegal desde Colombia ya que las poblaciones venezolanas de esta especie han sido severamente diezmadas. De este modo, la caza ilegal se ha desplazado hacia el Arauca, donde las poblaciones de capibaras han resultado fuertemente afectadas. Más recientemente, la cacería se ha desplazado hacia el Casanare, afectando a los municipios de Hato Corozal (el lugar objeto de estudio en este trabajo), Paz de Ariporo y Trinidad (Aldana-Domínguez et al. 2007). Por ejemplo, Higuera (2001) reportó que en marzo de 2003 se mataron 6800 capibaras en cuatro hatos del Casanare. Además, aproximadamente, entre el 38 y $44 \%$ de los ejemplares matados son hembras, lo cual es probablemente una tasa de extracción no sostenible en el tiempo (Payán 2007). De hecho en la zona del muestreo de la presente investigación, las densidades de capibaras son extremadamente más bajas (0.11-0.14 ind/ha) que en otras áreas del Casanare, como Paz de Ariporo (4.11-2.22 ind/ha) (Aldana-Domínguez \& Ángel-Escobar 2007). Esa menor densidad de capibaras en la zona de estudio no solo está determinada por la mayor presión de caza sino, también, porque las condiciones de hábitat son menos propicias y restrictivas en términos de agua, aunque, tan solo en 10 años, se cazaron en Paz de Ariporo unos 25000 ejemplares (Aldana-Domínguez et al. 2007). La situación de cuello de botella encontrada en este estudio difiere claramente de la expansión poblacional encontrada para dos poblaciones de capibaras estudiadas en el Gran Chaco paraguayo para 386 pares de bases de la región de control mitocondrial. Esas dos poblaciones mostraron una fuerte y rápida expansión poblacional además de flujo génico entre dos poblaciones previamente disjuntas (Campos-Krauer 2009, Campos-Krauer \& Wisely 2010). Por lo tanto, esa acción cinegética parece tener ya una clara repercusión en la estructura genética de esta especie, al menos, en ciertas áreas de los Llanos Orientales colombianos. De alguna manera, las autoridades colombianas y venezolanas pertinentes deberían formular políticas de protección y eliminación de la caza ilegal de esta especie para evitar esta pérdida acelerada de variabilidad genética que podría poner en serio riesgo la perdurabilidad de la misma en los ecosistemas de los Llanos Orientales.

\section{AGRADECIMIENTOS}

Agradecemos a la Universidad Nacional de Colombia y a IDEAWILD por el apoyo financiero de la fase de campo para la recolección de las muestras, a la Universidad Nacional Abierta y a Distancia por la financiación del proyecto, a Andrés Gutiérrez de la Fundación Arthur Stanley Gillow por el préstamo de las instalaciones del laboratorio de Biología Molecular para finalizar la fase de análisis de muestras y a la Pontificia Universidad Javeriana por ofrecer soporte logístico a M. R-G para llevar a cabo parte de los análisis genético poblacionales y escribir el presente manuscrito. 


\section{RESUMEN}

Los capibaras son los roedores más grandes del mundo, sin embargo, no se han realizado estudios genético poblacionales exhaustivos con ellos. En el presente trabajo se analizó la estructura genética de una manada de 31 capibaras (Hydrochoerus hydrochaeris) muestreada en Hato Corozal, Departamento de Casanare en los Llanos Orientales de Colombia, mediante cinco marcadores microsatelitales. La diversidad genética se determinó en 0.61 y un número promedio de alelos de 5.2, lo cual se puede considerar medio-bajo para este tipo de marcadores. De los cinco marcadores empleados, tres mostraron proporciones genotípicas en concordancia con lo esperado en equilibrio Hardy-Weinberg, mientras que un marcador mostró un exceso significativo de homocigotos y otro un exceso significativo de heterocigotos. No se encontraron diferencias significativas para esos cinco marcadores entre machos y hembras de la manada muestreada. La aplicación de diferentes procedimientos para detectar posibles cambios demográficos históricos (expansiones poblacionales o cuellos de botella) mostró claramente que la población analizada ha pasado por un cuello de botella extremadamente fuerte en épocas recientes. La limitada variabilidad genética encontrada y la fuerte evidencia de que la manada estudiada ha pasado por un cuello de botella reciente es probablemente el resultado de la cacería ilegal.

Palabras clave: capibara, chigüiro, microsatélites de ADN, estructura genética, cuello de botella, Llanos Orientales de Colombia.

\section{REFERENCIAS}

Acosta, L.H. 2010. Análisis del potencial de aprovechamiento de la capibara (Hydrochoeris hydrochoeris) en 6 comunidades chiquitanas del municipio de San Matías. Libro de Resúmenes del IX Congreso Internacional sobre Manejo de Fauna Silvestre en la Amazonía y America Latina. Santa Cruz de la Sierra, Bolivia.

Aldana-Domínguez, J., D.C. Ángel, M.I. Viera, C.E. Payán \& G. Ramírez. 2004. Uso y conservación del chigüiro (Hydrochaeris hydrochaeris) en el Casanare-Colombia. Libro de Resúmenes VI Congreso Internacional sobre Manejo de Fauna Silvestre en la Amazonía y Latinoamérica. Iquitos, Perú.

Aldana-Domínguez, J., J. Forero, J. Betancur \& J. Cavelier. 2002. Dinámica y estructura de la población de chigüiros (Hydrochaeris hydrochaeris: Rodentia, Hydrochaeridae) de Caño Limón, Arauca, Colombia. Caldasia 24: 445-458.

Aldana-Domínguez, J. \& D.C. Ángel-Escobar. 2007. Evaluación del tamaño y densidad de poblaciones silvestres de chigüiros en el Departamento del Casanare, p. 33-48. In J. Aldana-Domínguez, M.I. VieiraMuñoz \& D.C. Ángel-Escobar (eds.). Estudios sobre la ecología del chigüiro (Hydrochoerus hydrochaeris), enfocados a su manejo y uso sostenible en Colombia. Instituto Alexander von Humboldt, Bogotá D.C., Colombia.

Aldana-Domínguez, J., M.I. Vieira-Muñoz \& D.C. ÁngelEscobar. 2007. Estudios sobre la ecología del chigüiro (Hydrochoerus hydrochaeris), enfocados a su manejo y uso sostenible en Colombia. Instituto Alexander von Humboldt, Bogotá D.C., Colombia.

Allendorf, F.W. \& G. Luikart. 2007. Conservation and the genetics of populations. Blackwell, Massachusetts, EEUU.

Alho, C.J., Z.M. Campos \& H.C. Gonçalves. 1989. Ecology, social behavior and managment of the capybara (Hydrochaeris hydrochaeris) in the Pantanal of Brazil. Adv. Neotrop. Mammal. 163-194.

Álvarez, M., G. Cueto, R. Allekotte \& F. Kravetz. 1999. Cría de carpinchos en cautiverio: La experiencia argentina. Libro de resúmenes del IV Congreso Internacional sobre manejo de Fauna Silvestre en la Amazonía y Latinoamérica. Asunción, Paraguay.

Álvarez, M. \& F.O. Kravetz. 2004. Propuesta de aprovechamiento sustentable del carpincho (Hydrochoerus hydrochoeris, Rodentia) en Argentina. Libro de Resúmenes VI Congreso Internacional sobre Manejo de Fauna Silvestre en la Amazonía y Latinoamérica. Iquitos, Perú.

Arteaga, M.C. 2001. Aspectos relacionados con el uso del hábitat por parte del chigüiro (Hydrochaeris hydrochaeris) en el sector sur del Parque Nacional Amacayacu, trapecio amazónico colombiano. Libro de resúmenes del V Congreso Internacional de Manejo de Fauna Silvestre en Amazonía y Latinoamérica. Criterios de sostenibilidad. Cartagena de Indias, Colombia.

Atuesta-Dimian, N., A. Guzmán-Lenis, H.F. López-Arévalo \& P. Sánchez-Palomino. 2006. Evaluación de poblaciones silvestres de chigüiros (Hydrochoerus hydrochoeris) y sus hábitats en la Orinoquia colombiana. Libro de Resúmenes del VI Congresso Internacional sobre Manejo de Fauna Silvestre na Amazonia e América Latina. Ilhéus, Brasil.

Ballesteros, J. \& J.P. Jorgenson. 2009. Aspectos poblacionales del cacó (Hydrochoerus hydrochaeris isthmus) y amenazas para su conservación en el nor-occidente de Colombia. Mastozool. Neotrop. 16: 27-38. 
Bolkovic, M.L., R.D. Quintana, D. Ramadori, M. Elisetch \& J. Rabinovich. 2006. Proyecto Carpincho, p. 105119. In M.L Bolkovic \& D. Ramadori (eds.). Manejo de fauna silvestre en Argentina. Programa de uso sustentable. Dirección de Fauna Silvestre, Secretaría de Ambiente y Desarrollo Sustentable, Buenos Aires.

Botstein, D., R.L. White, M. Skolnick \& R.W. Davis. 1980. Construction of a genetic linkage in man using restriction fragment length polymorphism. Am. J. Hum. Genet. 32: 314-331.

Bruford, M.W. \& R.K. Wayne. 1993. Microsatellites and their application to population genetics studies. Curr. Opin. Genet. Dev. 3: 939-943.

Callen, D.F., A.D. Thompson, Y. Shen, H.A. Phillips, R.I. Richards, J.C. Mulley \& G.R. Sutherland. 1993. Incidence and origin of "null" alleles in the (AC)n microsatellite markers. Am. J. Hum. Genet. 52: 922-927.

Campos-Krauer, J.M. 2009. Landscape ecology of the capybara (Hydrochoerus hydrochoerys) in the Chaco region of Paraguay. Ph.D. Thesis, Kansas State University, EEUU.

Campos-Krauer, J.M. \& S.M. Wisely. 2010. Deforestation and cattle ranching drive rapid range expansión of capibara in the Gran Chaco ecosystem. Glob. Change Biol. 10: 1-13.

Cerqueira-Lopes, P.C., S. Siqueira Da Cunha Nogueira, C. Santana-Santos, A. mendes \& S.L. NogueiraFilho. 2006. Estudo da estructura social de capivaras (Hydrochoerus hydrochoeris) em caiveiro. Libro de Resúmenes del VI Congresso Internacional sobre Manejo de Fauna Silvestre na Amazonia e América Latina. Ilhéus, Brasil.

Chesser, R.K. 1991a. Gene diversity and female philopatry. Genetics 127: 437-447.

Chesser, R.K. 1991b. Influence of gene flow and breeding tactics on gene diversity within populations. Genetics 129: $573-583$.

Chesser, R.K., D.W. Sugg, O.E. Rhodes Jr, J.N. Novak \& M.H. Smith. 1993. Evolution of mammalian social structure. Acta Theriol. 38: 163-174.

Cockerham, C.C. 1973. Analyses of gene frequencies. Genetics 74: 679-700.

Cornuet, J.M. \& G. Luikart. 1996. Description and power analysis of two tests for detecting resent population bottleneck from alleles frequency data. Genetics 144 2001-2014.
Cossios, E.D., M. Lucherini, M. Ruiz-García \& B. Angers. 2010. Between high-altitude islands and high-altitude corridors. The population structure of the Andean cat (Leopardus jacobita). Anim. Conserv. in press.

Chiappero, M.B., A. Blanco, G.E. Calderón, M.S. Sabattini \& C.N. Gardenal. 2002. Genetic structure of populations of Calomys laucha (muridae, sigmodontinae) from central Argentina. Biochem. Syst. Ecol. 30: 1023-1036.

Chirhart, S.E., R.L. Honeycutt \& I.F. Greenbaum. 2005. Microsatellite variation and evolution in the Peromyscus maniculatus species group. Molec. Phylogenet. Evol. 34: 408-415.

Dallas, J.F, B. Dod, P. Boursot, E.M. Prager \& F. Bonhomme. 1995. Population subdivision and gene flow in Danish house mice. Mol. Ecol. 4: 311-320.

Dakin, E.E. \& J.C. Avise. 2004. Microsatellite null alleles in parentage analysis. Heredity 93: 504-509.

Dib, C., S. Faure \& C. Fizames. 1996. A comprehensive genetic map of the human genome base with 5.264 microsatellites. Nature 380: 152-154.

Di Rienzo, A., A. Peterson, J. Garza, A. Valdés, M. Slatkin \& N. Freimer. 1994. Mutational processes of simplesequence repeat loci in human populations. P. Natl. Acad. Sci. USA 91: 3166-3170.

Dobson, F.S. \& B. Zinner. 1998. Social groups, genetic structure, and conservation. In M. Festa-Bianchet \& M. Apoollonio. (eds.). 2003. Animal Conservation and Wildlife conservation. Island, Washington, DC, EEUU.

Efron, B. \& R.J. Tibshirani. 1993. An introduction to the bootstrap. Chapman and Hall, Nueva York, EEUU

Emmons, H.L. \& H. Feer. 1997. Neotropical Rainforest Mammals. A Field Guide. University of Chicago, Chicago, EEUU.

Goldman, E.A. 1912. New mammals from eastern Panama. Smithson. Misc. Collect. 60: 1-18.

Goncalves-Saraiva, D. \& G.F. Da Silva Rocha. 2006. Estudo populacional de Hydrochaerus hydrochaeris no Rio Cipó-Jaboticatubas (MG). Libro de Resúmenes del VI Congresso Internacional sobre Manejo de Fauna Silvestre na Amazonia e América Latina. Ilhéus, Brasil.

González, J. 2001. Aprovechamiento sostenible de la producción de biorecursos (heliconias, huevos de charapa, miel de abeja y chigüiros) por pequeños productores en la región de influencia del río Caguán, 
Caquetá, Colombia. Libro de resúmenes del V Congreso Internacional de Manejo de Fauna Silvestre en Amazonía y Latinoamérica. Criterios de sostenibilidad. Cartagena de Indias, Colombia.

González-Jiménez, E. 1995. El capibara (Hydrochaerus hydrochaeris). Estado actual de su producción. Serie FAO: Producción y Sanidad Animal, Roma.

Guzmán-Lenis, A.R., H.F. López-Arévalo \& P. SánchezPalomino. 2006. Evaluación del hábitat disponible para el chigüiro Hydrochoerus hydrochoeris (Linnaeus, 1766) en las sabanas inundables de la Orinoquía colombiana. Libro de Resúmenes del VI Congresso Internacional sobre Manejo de Fauna Silvestre na Amazonia e América Latina. Ilhéus, Brasil.

Harrison-Matthews, L. 1977. La vida de los Mamíferos II. Destino, Barcelona, España.

Hernández-Camacho, J., J.E. Pachón \& J.V. Rodríguez. 1983. Evaluación de las poblaciones de chigüiro (Hydrochaeris hydrochaeris) en los hatos Brasilia, Guamito, La Aurora, La Borra, El Danubio, La Veremos y Mapurisa, municipio de Hato Corozal, Casanare. Informe presentado a Instituto Nacional de los Recursos Naturales Renovables y del AmbienteINDERENA. Bogotá, Colombia.

Herrera, E. 1999. Comportamiento, conservación y manejo de fauna Silvestre: el caso del capibara en Venezuela. Etología 7: 41-46.

Herrera, E.A., M.E. Chemello, E.A. Lacey, V. Sala \& B.F. Sousa. 2004. Characterization of microsatellite markers from capybaras, Hydrochoerus hydrochaeris (Rodentia: Hydrochoeridae). Mol. Ecol. Notes 4: 541-543.

Herrera, E. \& D. Macdonald. 1989. Resource utilization and territoriality in group-living capybaras (Hydrochaeris hydrochaeris). J. Anim. Ecol. 58: 667-679.

Higuera, C. 2001. Condena por la masacre de 10000 chigüiros. El Espectador, 12 de julio, Bogotá.

ICN. Instituto de Ciencias Naturales. 2002. Implementación de la fase inicial del programa del manejo del chigüiro (Hydrochaeris hydrochaeris) en semicautiverio como estrategia de conservación de la biodiversidad en la Orinoquía colombiana y acopio de información complementaria. Universidad Nacional de Colombia, Ministerio de Medio Ambiente, Vivienda y Desarrollo Territorial, Bogotá, Colombia.

Issa Rickli, R., N.R. Dos Reis \& N. Kishino. 2008. Atividades e locais ocupados por capivaras- Hydrocoerus hydrochaeris- (Rodentia: Caviidae) em um fragmento florestal (85,47 ha) em Londrina, norte do Paraná,
Brasil. Livro de Resumenes do XXVII Congresso Brasileiro de Zoología. EXPOTRADE. Curitiba, Paraná, Brasil.

Kimmel, M., R. Chakraborty, J.P. King, M. Bamshad, W.S. Watkins \& L.B. Jorde. 1998. Signatures of population expansion in microsatellite repeat data. Genetics 148 : 1921-1930.

Lozada-Perdomo, P.A. 2007. Análisis del mercado nacional e internacional para productos derivados del chigüiro, p. 145-182. In J. Aldana-Domínguez, M.I. Vieira-Muñoz \& D.C. Ángel-Escobar (eds.). Estudios sobre la ecología del chigüiro (Hydrochoerus hydrochaeris), enfocados a su manejo y uso sostenible en Colombia. Instituto Alexander von Humboldt, Bogotá D.C., Colombia.

Luikart, G., F. Allendorf, B. Sherwin \& J.M. Cornuet. 1998. Distortion of allele frequency distribution provides a test for recent population bottleneck. J. Hered. 86: 319-322.

Maldonado-Chaparro, A.A., A. Guzmán-Lenis, H.F. LópezArevalo, P. Sánchez-Palomino \& O.L. MontenegroDiaz. 2006. Modelo para la evaluación de la calidad de hábitat disponible para el chigüiro (Hydrochoerus hydrochoeris) en la sabana inundable de la Orinoquía de Colombia. Libro de Resúmenes del VI Congresso Internacional sobre Manejo de Fauna Silvestre na Amazonia e América Latina. Ilhéus, Brasil.

Maldonado-Chaparro, A.A. \& P. Sánchez. 2010. Spatio-temporal patterns of capybara (Hydrochoerus hydrochaeris) population in the flooded savannas of Colombia. Mastozool. Neotrop. en prensa.

Marshall, T.C., J. Slate, E.B. Kruuk \& J.M. Pemberton. 1998. Statistical confidence for likelihood-based paternity inference in natural populations. Mol. Ecol. 7: 639-655.

McConnell, S.K.J., D.E. Ruzzante, P.T. O’Reilly, L. Hamilton \& J.M. Wright. 1997. Microsatellite loci revealed highly significant genetic differentiation among Atlantic salmon (Salmo salar L.) stocks from the east coast of Canada. Mol. Ecol. 6: 1075-1089.

Mesa, E. \& J. Sánchez. 2002. Implementación de la fase inicial del programa de manejo del chigüiro (Hydrochaeris hydrochaeris) en semicautiverio, como estrategia de conservaciónde la biodiversidad en la orinoquía colombiana y acopio de información complementaria. Convenio de cooperación científica y tecnológica No. 085. Ministerio del Medio Ambiente - Universidad Nacional de Colombia, Bogotá, Colombia. 
Mesa-González, E., H.F. López-Arevalo \& P. SánchezPalomino. 2006. Simulación del efecto de la cosecha en poblaciones silvestres de chigüiros Hydrochoerus hydrochaeris en la Orinoquia colombiana. Libro de Resúmenes del VI Congresso Internacional sobre Manejo de Fauna Silvestre na Amazonia e América Latina. Ilhéus, Brasil.

Moreira, J.R. 1993. O manejo da capivara (Hydrochaeris hydrochaeris). En Resúmenes del I Congreso Internacional sobre manejo de Fauna Silvestre en la Amazonía y Latinoamérica. Manaus, Brasil.

Mulligan, M., J. Andrew, B. Sophia \& J. Aldana-Domínguez. 2007. Aplicación de la fotografía aérea digital y la videografía para la estimación de las poblacionales del chigüiro en los Llanos Orientales de Colombia, Departamento del Casanare, p. 49-67. In J. Aldana-Domínguez, M.I. Vieira-Muñoz \& D.C. Ángel-Escobar (eds.). Estudios sobre la ecología del chigüiro (Hydrochoerus hydrochaeris), enfocados a su manejo y uso sostenible en Colombia. Instituto Alexander von Humboldt, Bogotá D.C., Colombia.

Nei, M., T. Maruyama \& R. Chakraborty. 1975. The bottleneck effect and genetic variability in populations. Evolution 29: 1-10.

Nogueira-Filho, S.L. \& S.S.C. Nogueira. 2004. Captive breeding programs as an alternative for wildlife conservation in Brazil, p. 171-190. In S. Kirsten, J.M.V. Fragoso \& E.R. Bodmer (eds.). People in Nature: Wildlife Management and conservation in Latin America. Columbia University, Nueva York, EEUU.

Nowak, R.M. 1991. Walker's mammals of the World. The Johns Hopkins University, Baltimore, EEUU.

Ohta, T. \& M. Kimura. 1973. A model of mutation appropriated to estimate the number of electrophoretically detectable alleles in a finite population. Genet. Res. 22: 201-204.

Ojasti, J. 1973. Estudio biológico del chigüire o capibara. Fondo Nacional de Investigaciones Agropecuarias, Caracas, Venezuela.

Ojasti, J. 1980. Ecology of capibara raising on inundated savannas of Venezuela. Trop. Ecol. Develop. 1980: 287-293.

Ojasti, J. 1991. Human exploitation of capybara, p. 236253. In J.G. Robinson \& K.H. Redford (eds.). Neotropical wildlife use and conservation. Chicago University, Chicago, EEUU.

Paetkau, D., W. Calvert, I. Stirling \& C. Strobeck. 1995. Microsatellite analysis of population structure in Canadian polar bears. Mol. Ecol. 4: 347-354.
Pardo, B.G., M. Hermida, C. Fernandez, C. Bouza, M. Perez, A. Llavona, L. Sanchez \& P. Martinez. 2006. A set of highly polymorphic microsatellites useful for kinship and population analysis in turbot (Scophthalmus maximus L.). Aquac. Res. 37: 1578-1582.

Patiño, A.F., E.A. Prada \& L.E. Martínez. 2001. Estimación poblacional de Hydrochaeris hydrochaeris isthmus en un humedal de San Marcos, Sucre, Colombia. Libro de resúmenes del V Congreso Internacional de Manejo de Fauna Silvestre en Amazonía y Latinoamérica. Criterios de sostenibilidad. Cartagena de Indias, Colombia.

Payán, E. 2007. Análisis de proporciones de edad y sexo a partir de cráneos resultantes de cosechas de chigüiros en los Llanos Orientales de Colombia, Departamento del Casanare, para el uso y conservación de la especie, p. 85-103. In J. Aldana-Domínguez, M.I. Vieira-Muñoz \& D.C. Ángel-Escobar (eds.). Estudios sobre la ecología del chigüiro (Hydrochoerus hydrochaeris), enfocados a su manejo y uso sostenible en Colombia. Instituto Alexander von Humboldt, Bogotá D.C., Colombia.

Peceño, M.C. 1983. Estudio citogenético y genético evolutivo del chigüire género Hydrochaeris. Trabajo Especial de Grado, Universidad Simón Bolívar, Caracas.

Pinheiro-Bartha, M.M., F.E. Rodrigues Da Silva, H.L. Tavares-Dias \& A. Lopes-Morais. 2006. Análise do risco microbiológico do consumo de carne salgada de jacaré (Caiman sp.) e de capivara (Hydrochoerus hydrochoeris) comercializadas no mucicipio de Abaetetuba-Pará. Libro de Resúmenes del VI Congresso Internacional sobre Manejo de Fauna Silvestre na Amazonia e América Latina. Ilhéus, Brasil.

Piry, S., G. Luikart \& J.M. Cornuet. 1999. Bottleneck: a computer program for detecting recent reductions in the effective population size using allele frequency data. J. Hered. 90: 502-503.

Pope, T. 1992. The influence of dispersal patterns and mating system on genetic differentiation within and between populations of the red howler monkey (Alouatta seniculus). Evolution 46: 1112-1128.

Prada, E.A. \& L.E. Martínez. 2001. Estimación poblacional de Hydrochaeris hidrochaeris isthmius en Sucre, Colombia. Libro de resúmenes del V Congreso Internacional de Manejo de Fauna Silvestre en Amazonía y Latinoamérica. Criterios de sostenibilidad. Cartagena de Indias, Colombia.

Raymond, M. \& F. Rousset. 1995. GENEPOP (version 1.2): population genetics software for exact tests and ecumenicism. J. Hered. 86: 248-249. 
Reich, D.E. \& D.B. Goldstein. 1998. Genetic evidence for a Paleolitic human population expansion in Africa. Proc. Natl. Acad. Sci. USA 95: 8119-8123.

Reich, D.E., M.W. Feldman \& D.B. Goldstein. 1999. Statistical properties of two tests that use multilocus data sets to detect population expansions. Mol. Biol. Evol. 16: 453-466.

Robertson, A. \& W.G. Hill. 1984. Deviations from HardyWeinberg proportions, sampling variances and use in estimation of inbreeding coefficients. Genetics 107: 703-718.

Ruiz-García, M. 1991. Más sobre genética de poblaciones de Felis catus en la costa Mediterránea Española: Un análisis de la Estructura Genética de las poblaciones naturales de gatos. Evol. Biol. 5: 227-283.

Ruiz-García, M. 1993. Analysis of the Evolution and Genetic Diversity within and between Balearic and Iberian cat populations. J. Hered. 84: 173-180.

Ruiz-García, M. 1998. Genetic structure and evolution of different cat populations (Felis catus) in Spain, Italy, Argentina at microgeographical level. Acta Theriol. 43: 39-66.

Ruiz-García, M. 2001. Diversidad genética como herramienta de zonificación ambiental: Estudios moleculares (microsatélites) en el caso de Primates y Félidos neotropicales comportan una nueva perspectiva, $\mathrm{p}$. 85-108. In T. Defler \& P.A. Palacios (eds.). Zonificación Ambiental para el Ordenamiento Territorial en la Amazonía Colombiana. Universidad Nacional de Colombia, Bogotá.

Ruiz-García, M. 2010a. Micro-geographical genetic structure of Inia geoffrensis in the Napo-Curaray river basin by means of Chesser's models, p. 131-160. In M. RuizGarcía \& J. Shostell (eds.). "Biology, Evolution, and Conservation of River Dolphins Within South America and Asia”. Nova Science, Nueva York, EEUU.

Ruiz-García, M. 2010b. Changes in the demographic trends of pink river dolphins (Inia) at the microgeographical level in Peruvian and Bolivian rivers and within the Upper Amazon: Microsatellites and mtDNA analyses and insights into Inia's origin, p. 161-192. In. M. Ruiz-García \& J. Shostell (eds.). "Biology, evolution, and conservation of River Dolphins Within South America and Asia". Nova Science, Nueva York, EEUU.
Ruiz-García, M. 2010c. The genetic demography history and phylogeography of the Andean bear (Tremarctos ornatus; Ursidae) by means of microsatellites and mtDNA markers. In M. Ruiz-García \& J. Shostell (eds.). "Molecular Population Genetics, Phylogenetics, Evolutionary Biology and Conservation of the Neotropical Carnivores". Nova Science, Nueva York, EEUU.

Ruiz-García, M., P. Orozco-terWengel, A. Castellanos \& L. Arias. 2005. Microsatellite analysis of the spectacled bear (Tremarctos ornatus) across its range distribution. Genes Genet. Syst. 80: 57-69.

Ruiz-García, M., E. Payán, A. Murillo \& D. Álvarez. 2006. DNA Microsatellite characterization of the Jaguar (Panthera onca) in Colombia. Genes Genet. Syst. 81: 115-127.

Ruiz-García, M., M. Martínez-Agüero, D. Álvarez \& S. Goodman. 2009. Variabilidad genética en géneros de Ciervos neotropicales (Mammalia: Cervidae) según loci microsatélitales. Rev. Biol. Trop. 57: 879-904.

Schivo, F., P. Kandus, P. Minotti \& R.D. Quintana. 2010. Determinación de áreas con valor conservativo para el carpincho (Hydrochoerus hydrochaeris) a escala regional mediante la aplicación de un modelo de aptitud potencial de hábitat. Libro de Resúmenes del IX Congreso Internacional sobre Manejo de Fauna Silvestre en la Amazonía y America Latina. Santa Cruz de la Sierra, Bolivia.

Siqueira da Cunha Nogueira, S. \& S.L.G. NogueiraFilho. 1997. Determinacao das causas de infanticidio en grupos de capivara (Hydrochoerus hydrochoeris hydrochoeris) criadas em cativeiro. Libro de Resúmenes del III Congreso Internacional sobre Manejo de Fauna Silvestre en la Amazonía y Latinoamérica. Santa Cruz de la Sierra, Bolivia.

Soini, P. \& M. Soini. 1992. Ecología del ronsoco o capibara (Hydrochaeris hydrochaeris) en la reserva nacional Pacaya-Samiria, Perú. Folia Amaz. 4: 119-133.

Storz, J.F. 1999. Genetic consequences of mammalian social structure. J. Mammal. 80: 553-569.

Sugg, D.W., R.K. Chesser, F.S. Dobson \& J.L. Hoogland. 1996. Population genetics meets behavioral ecology. Trends Ecol. Evol. 11: 338-342. 
Trapido, H. 1949. Gestation period, young, and maximum weight of the isthmian capibara, Hydrocherus isthmius Goldman. J. Mammal. 30: 433-437.

Vega, R. 2006. Estructura y diversidad genética de Oryzomys palustris cozumelae de la isla de Cozumel. Tesis de maestría, Instituto de Ecología, UNAM, México.

Vieira-Muñoz, M. I. 2007. Identificación y caracterización de los ecosistemas asociados a las poblaciones de chigüiros (Hydrochoerus hydrochaeris) y su relación con el comportamiento en el Casanare, Colombia, p. 105-128. In J. Aldana-Domínguez, M.I. VieiraMuñoz \& D.C. Ángel-Escobar (eds.). Estudios sobre la ecología del chigüiro (Hydrochoerus hydrochaeris), enfocados a su manejo y uso sostenible en Colombia. Instituto Alexander von Humboldt, Bogotá D.C., Colombia.

Weber, J.L. \& C. Wong. 1993. Mutation of human short tandem repeats. Hum. Mol. Genet. 2: 1123-1128.
Weir, B.S. \& C.C. Cockerham. 1984. Estimating F-statistics for the analysis of population structure. Evolution 38: 1358-1370.

Whatman. FTA ${ }^{\circledR}$ protocols: collect transport, archive and access nucleic acids all at room temperature 2002 (disponible en línea: www.cosmobio.com.ar/docs/ fta\%20protocols.pdf).

Wilson, D.E. \& D.M. Reeder. 2005. Mammal species of the World. A taxonomic and geographic reference. Johns Hopkins University, Baltimore, EEUU.

Zhivotovsky, L.A. \& M.W. Feldman. 1995. Microsatellite variability and genetic distances. Proc. Natl. Acad. Sci. USA 92: 11549-11552.

Zhivotovsky, L.A., L. Bennett, A.M. Bowcock \& M.W. Feldman. 2000. Human population expansion and microsatellite variation. Mol. Biol. Evol. 17: $757-767$. 
